02

\title{
Плотность энергетического спектра электрона в поле изображения и запирающем электрическом поле
}

\author{
(C) П.А. Головинский, М.А. Преображенский, А.А. Дробышев \\ Воронежский государственный технический университет, лаборатория фризических исследований, \\ 394006 Воронеж, Россия \\ e-mail: golovinski@bk.ru
}

Поступила в редакцию 24.09.2020 г.

В окончательной редакции 24.09.2020 г.

Принята к публикации 03.10.2020 г.

В квазиклассическом приближении описана плотность спектра энергий электрона вблизи поверхности металла, связанного полем изображения и запирающим электростатическим полем. В рассматриваемой системе реализуется механизм конфайнмента, и энергетический спектр для движения электрона в направлении, перпендикулярном поверхности металла, является полностью дискретным. Плотность энергетического спектра выражена через эллиптические интегралы, аргумент которых является сигмоидальной функцией, переходящей при выключении поля в ступенчатую функцию Хевисайда. Введен безразмерный энергетический параметр, определяющий интервалы с качественно различным изменением ширины классически доступной области движения. При больших положительных значениях энергетического параметра плотность спектра асимптотически стремится к плотности энергий в треугольном потенциале с добавлением кулоновской логарифмической поправки, а при отрицательных значениях энергетического параметра плотность спектра переходит в зависимость, справедливую для одномерного кулоновского потенциала. Получены приближенные выражения для плотности спектра через элементарные функции в широком диапазоне энергий электрона и напряженности электрического поля.

Ключевые слова: потенциал изображения, плотность спектра, запирающий потенциал, состояния вблизи поверхности металла.

DOI: $10.21883 /$ OS.2021.02.50553.242-20

\section{Введение}

Вблизи поверхности проводника заряд испытывает электростатическое притяжение к своему изображению. В возникающем за счет этого одномерном потенциале, близком по характеристикам к кулоновскому взаимодействию, образуются связанные состояния с ридберговским спектром энергий: $E_{n}=-1 / 32 n^{2}$ a.u., $n=1,2, \ldots, \infty[1]$. Они проявляются экспериментально в резонансах туннельного тока [2] и спектрах фотоэмиссии [3-6]. Связанные локализованные приповерхностные состояния могут модифицировать отклик наносистемы на внешнее воздействие, вследствие чего развитие наноструктурных исследований стимулирует учет влияния состояния электронов, связанных потенциалом изображения, на различные поверхностные эффекты [7-10].

Ранее на основе теории возмущений было исследовано поведение электрона, связанного потенциалом изображения, во внешних электрическом и магнитном полях [11]. В рамках теории возмущений были получены выражения для вероятностей связанно-связанных и связанно-свободных вынужденных переходов в слабом электромагнитном поле [12]. Однако современные технологии генерации терагерцовых униполярных импульсов позволяют создавать сильные квазистатические поля с напряженностью более $1 \mathrm{MV} / \mathrm{cm}[13,14]$, для которых применение теории возмущений не соответствует физическим условиям. В таких полях для описания взаимодействия электрона, связанного полем изображения и электрическим полем, можно следовать подходу, основанному на применении квазиклассического приближения, используемого для расчета эффекта Штарка в атоме водорода [15]. Этим методом ранее были получены волновые функции и спектр квазистационарных состояний электрона в потенциале изображения при наложении постоянного разрушающего электрического поля [16].

Поведение электрона, связанного полем изображения и запирающим электрическим полем, не имеет аналога в трехмерной задаче. Возникающий при этом механизм одномерного конфайнмента электрона определяет плотность дискретного спектра состояний. Пространственная ширина области локализации электрона задается его энергией и напряженностью внешнего поля. Проведенные ранее квазиклассические расчеты энергетического спектра электрона в таких условиях были выполнены лишь для малой напряженности внешнего поля [17] в области отрицательных энергий. Целью данной работы является описание плотности спектра связанных состояний электрона в потенциале изображения для широкого интервала напряженности внешнего электрического поля и энергий электрона. 


\section{Основные уравнения для квазиклассической плотности спектра}

Потенциальная энергия электрона в поле изображения и перпендикулярном поверхности металла запирающем однородном электрическом поле имеет вид

$$
U(z)=F z-\frac{1}{4 z}
$$

Направление оси $O Z$ выбрано перпендикулярно поверхности металла. Слагаемое $F z$ описывает энергию взаимодействия электрона с однородным электрическим полем напряженностью $F$, слагаемое $-1 / 4 z$ определяет потенциальную энергию взаимодействия электрона с полем изображения идеального проводника.

Пространственные переменные в уравнении Шредингера в данной задаче разделяются. Движение, параллельное поверхности, является свободным, а спектр энергий этого движения, задаваемый соотношением $E_{x y}=p_{x}^{2} / 2+p_{y}^{2} / 2$, полностью непрерывным. Соответствующие компоненты волновой функции описываются плоскими волнами $\exp \left(i p_{x} x\right)$ и $\exp \left(i p_{y} y\right)$. Движение электрона в направлении, перпендикулярном поверхности металла, ограниченное значениями $z>0$, описывается волновой функцией $\varphi(z)$, являющейся решением одномерного уравнения Шредингера:

$$
\frac{d^{2} \varphi}{d z^{2}}+2\left(E_{z}-F z+\frac{1}{4 z}\right) \varphi(z)=0
$$

При положительных значениях напряженности и любых энергиях электрона движение в потенциале (1) является финитным, вследствие чего энергетический спектр становится полностью дискретным. Функция

$$
p(z)=\sqrt{2\left(E_{z}-F z+1 / 4 z\right)}
$$

в классически доступной области движения $0 \leq z \leq z_{1}$ совпадает с нормальной к поверхности металла компонентой импульса электрона [17]. Ширина области классически допустимого движения определяется положительным корнем уравнения

$$
4 F z^{2}-4 E_{z} z-1=0,
$$

Корни уравнения (4) есть

$$
z_{1,2}=\frac{1}{2 \sqrt{F}}\left(\varepsilon \pm \sqrt{\varepsilon^{2}+1}\right)
$$

где введен энергетический параметр

$$
\varepsilon=\frac{E_{z}}{\sqrt{F}}
$$

График зависимости ширины классически доступной области движения от параметра $\varepsilon$ приведен на рис. 1.

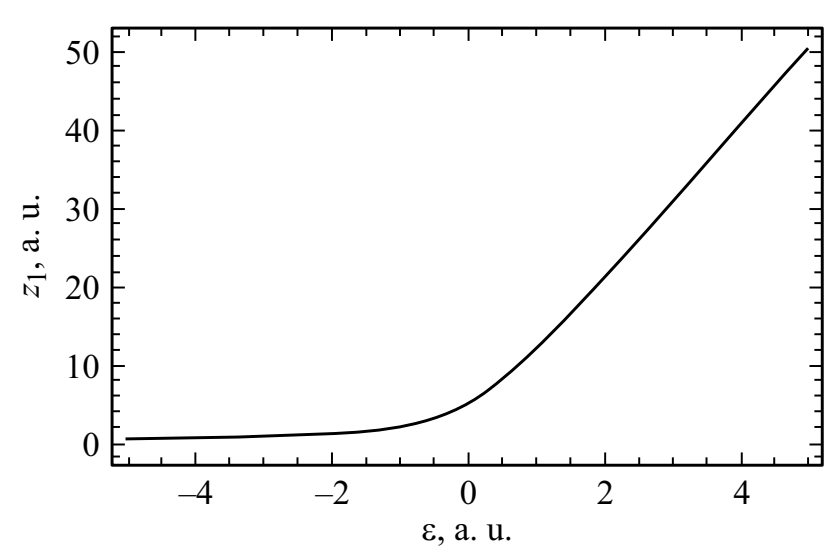

Рис. 1. Зависимость положения точки поворота $z_{1}$ от $\varepsilon$ в поле напряженностью $F=0.01$ a.u.

Квазиклассическое условие квантования энергии для одномерного кулоновского потенциала имеет вид [18]

$$
\int_{0}^{z_{1}} p(z) d z=\pi n
$$

Дифференцирование соотношения (7) по энергии дает следующее выражение для плотности состояний:

$$
\rho\left(E_{z}\right)=\frac{d n}{d E_{z}}=\frac{1}{\pi} \int_{0}^{z_{1}} \frac{d z}{p(z)} .
$$

Поведение ширины области классически доступного движения и плотности энергетического спектра в зависимости от энергетического параметра качественно различны в трех интервалах его значений. При выполнении условия $|\varepsilon|>\sqrt{2}$ ширину области классически доступного движения можно представить в виде разложения по обратным значениям параметра $\varepsilon$ в виде

$$
z_{1} \approx \frac{1}{\sqrt{F}}\left(\frac{\varepsilon+|\varepsilon|}{2}+\frac{1}{4|\varepsilon|}\left(1-\frac{1}{4 \varepsilon^{2}}+\frac{1}{8 \varepsilon^{4}}\right)\right) .
$$

В соответствии с разложением (9) при больших положительных значениях $\varepsilon$ ширина классически доступной области задается условием $z_{1} \approx \varepsilon / \sqrt{F}$. Физически это соответствует действию только электрического поля при наличии ограничивающей стенки и пренебрежению действием кулоновского поля. В этом пределе справедливой становится модель треугольного потенциала [19], в которой плотность состояний растет с увеличением энергии по закону

$$
\rho(\varepsilon)=\frac{\sqrt{2 \varepsilon}}{\pi F^{1 / 4}} .
$$

При больших отрицательных значениях $\varepsilon$ в соответствии с формулой (9) величина $z_{1}$ обратно пропорциональна параметру $4|\varepsilon| \sqrt{F}$, что физически соответствует пренебрежению влиянием электрического поля и 
действию лишь одномерного кулоновского потенциала. В этом пределе спектр энергий электрона является ридберговским с плотностью состояний

$$
\rho(\varepsilon)=\frac{1}{\pi 2^{-5 / 2} F^{1 / 4}|\varepsilon|^{3 / 2}} .
$$

При малых значениях параметра $|\varepsilon|<\sqrt{2}-1$ ширина области классически доступного движения $z_{1}$ зависит от параметра $\varepsilon$ по закону

$$
z_{1} \approx \frac{1+\varepsilon+\varepsilon^{2} / 2-\varepsilon^{4} / 8}{2 \sqrt{F}} .
$$

В интервалах значений $\sqrt{2}>|\varepsilon|>\sqrt{2}-1$ зависимость ширины от энергетического параметра описывается разложением

$$
\begin{aligned}
z_{1} \approx & \frac{1}{\sqrt{2 F}}\left(1+\frac{\varepsilon}{\sqrt{2}}+\frac{\left(\varepsilon^{2}-1\right)}{4}-\frac{\left(\varepsilon^{2}-1\right)^{2}}{32}\right. \\
& \left.+\frac{\left(\varepsilon^{2}-1\right)^{3}}{128}-\frac{5\left(\varepsilon^{2}-1\right)^{4}}{2048}\right) .
\end{aligned}
$$

Разложения (9), (12) и (13) позволяют описать изменение ширины области классически доступного движения с относительной погрешностью, не превышающей $10^{-3}$.

В дискретном спектре операция дифференцирования в уравнении (8) приводит к выражению для плотности спектра лишь при высокой плотности уровней $\rho(\varepsilon) \gg 1$, что определяет нижнюю границу энергетического параметра. Для отрицательных значений $\varepsilon$ в соответствии с уравнением (11) условие высокой плотности принимает вид

$$
|\varepsilon| \ll \frac{1}{2 \pi^{2} F^{1 / 2}} .
$$

Выразив импульс (4) через корни уравнения (5) в виде

$$
p(z)=\sqrt{2 F} \frac{\sqrt{\left(z-z_{1}\right)\left(z_{2}-z\right)}}{\sqrt{z}}
$$

и подставив это выражение в формулу (8), получим плотность спектра в виде

$$
\rho\left(E_{z}\right)=\frac{1}{\pi \sqrt{2 F}} \int_{0}^{z_{1}} \frac{\sqrt{z} d z}{\sqrt{\left(z-z_{1}\right)\left(z_{2}-z\right)}} .
$$

Плотность состояний (16) выражается [20] через полные эллиптические интегралы

$$
I_{\mu}(q)=\int_{0}^{\pi / 2}\left(1-q^{2} \sin ^{2} x\right)^{\mu} d x
$$

в виде

$$
\begin{aligned}
\rho(\varepsilon, F) & =\frac{\sqrt{2}\left[\left(z_{1}-z_{2}\right) I_{1 / 2}(h)+z_{2} I_{-1 / 2}(h)\right]}{\pi \sqrt{F} \sqrt{z_{1}-z_{2}}} \\
& =\frac{\sqrt{2} \tilde{\rho}(h)\left(\varepsilon^{2}+1\right)^{1 / 4}}{\pi F^{3 / 4}},
\end{aligned}
$$

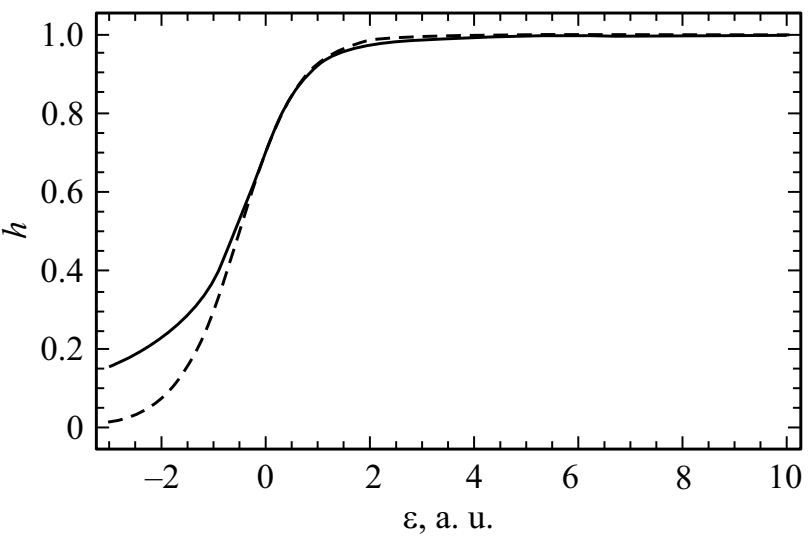

Рис. 2. Зависимость ступенчатой функции $h$ и аппроксимации Ферми-Дирака от энергетического параметра $\varepsilon$ : сплошная линия - точное значение (19), штриховая линия - аппроксимация (22).

где аргумент эллиптических интегралов

$$
h=\sqrt{\frac{z_{1}}{z_{1}-z_{2}}}=\sqrt{\frac{\varepsilon+\sqrt{\varepsilon^{2}+1}}{2 \sqrt{\varepsilon^{2}+1}}}
$$

является функцией параметра $\varepsilon$. В уравнении (18) для удобства введена приведенная плотность спектра

$$
\tilde{\rho}(h)=I_{1 / 2}(h)+\left(h^{2}-1\right) I_{-1 / 2}(h),
$$

зависящая только от безразмерного энергетического параметра.

\section{Результаты расчета плотности спектра и анализ его асимптотического поведения}

График зависимости функции $h$ от энергетического параметра приведен на рис. 2. Ширина интервала изменения сигмоидальной функции $h(\varepsilon)$ определяется соотношением $\Delta \varepsilon \sim 1$. В соответствии с формулой (6) в пределе слабого поля ширина интервала энергии электрона, в котором функция $h(\varepsilon)$ изменяется от нуля до единицы, уменьшается по закону $\Delta E_{z}=\Delta \varepsilon \sqrt{F}$. При выключении поля функция $h(\varepsilon)$ переходит в функцию Хевисайда $\theta(\varepsilon)$. Как показывают расчеты, главная часть зависимости $h(\varepsilon)$ может быть представлена в виде функции Ферми-Дирака

$$
\tilde{h}(\varepsilon)=\frac{1}{1+\alpha \exp (-\kappa \varepsilon)} .
$$

Определив константы $\alpha$ и $\kappa$ из условий равенства логарифмических производных функций (19) и (21) в точке $\varepsilon=0$, получим

$$
\tilde{h}(\varepsilon)=\left(1+(\sqrt{2}-1) \exp \left(\frac{\varepsilon}{\sqrt{2}-2}\right)\right)^{-1} .
$$




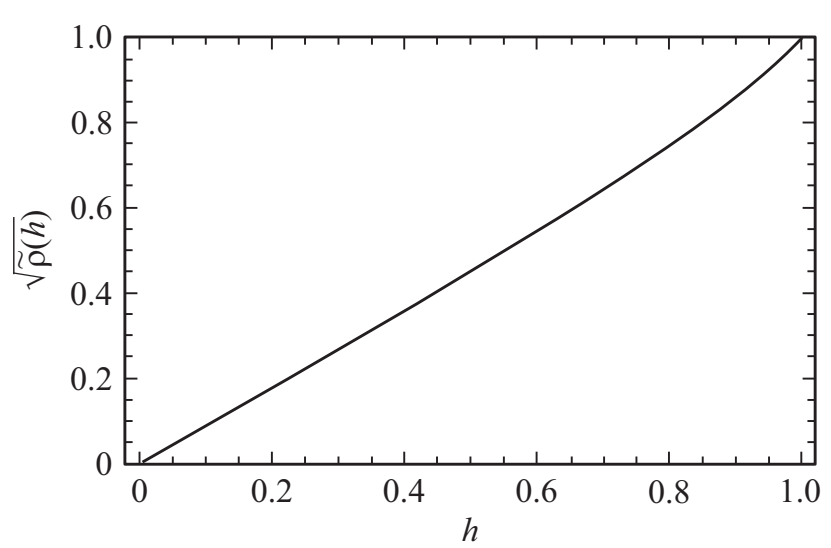

Рис. 3. Зависимость $\sqrt{\tilde{\rho}(h)}$ от параметра $h$.

Как видно из рис. 2, аппроксимация Ферми-Дирака хорошо воспроизводит зависимость при положительных значениях энергетического параметра и нуждается в уточнении в области $\varepsilon \leq-1$. Для этого представим функцию $h(\varepsilon)$ в виде

$$
\begin{aligned}
h(\varepsilon)= & \left(1+(\sqrt{2}-1) \exp \left(\frac{\varepsilon}{\sqrt{2}-2}\right)\right)^{-1} \\
& +\frac{1-\operatorname{sign}(\varepsilon)}{2} \Delta h .
\end{aligned}
$$

Раскладывая разность функций (19) и (22) в степенной ряд по энергетическому параметру, можно найти искомую поправку $\Delta h(\varepsilon)$ к функции $\tilde{h}(\varepsilon)$. Полученные рациональные разложения довольно громоздки. Десятичное представление поправки $\Delta h$ с точностью до членов $\sim \varepsilon^{3}$ имеет вид

$$
\begin{aligned}
\Delta h(\varepsilon) & =0.00211493+0.050236 \varepsilon \\
& +0.197941 \varepsilon^{2}+0.071684 \varepsilon^{3} .
\end{aligned}
$$

Функция (23) с учетом поправки в виде (24) обеспечивает относительную точность аппроксимации функции $h(\varepsilon)$ в интервале $-2<\varepsilon<\infty$ не хуже $10^{-4}$. На рис. 2 в окрестности нулевой энергии видна область зависимости $h(\varepsilon)$, близкой к линейной и описываемой отрезком разложения в ряд по положительным степеням энергетического параметра в виде

$$
h(\varepsilon) \approx \frac{1}{\sqrt{2}}\left(1+\frac{\varepsilon}{2}-\frac{\varepsilon^{2}}{8}-\frac{3 \varepsilon^{3}}{16}+\frac{11 \varepsilon^{4}}{128}\right) .
$$

В пределе больших отрицательных значений энергетического параметра поведение функции $h(\varepsilon)$ дается асимптотическим разложением

$$
h(\varepsilon) \approx \frac{1}{2|\varepsilon|}\left(1-\frac{3}{8 \varepsilon^{2}}+\frac{31}{128 \varepsilon^{4}}-\frac{187}{1024 \varepsilon^{6}}\right) .
$$

В области $|\varepsilon|>0$ наблюдается быстрый выход зависимости $h(\varepsilon)$ на асимптотическое значение $h \approx 1$, описываемое разложением

$$
h(\varepsilon) \approx 1-\frac{1}{8 \varepsilon^{2}}+\frac{11}{128 \varepsilon^{4}}-\frac{69}{1024 \varepsilon^{6}} .
$$

Определим теперь зависимость приведенной плотности от функции $h$. График зависимости функции $\sqrt{\tilde{\rho}(h)}$ приведен на рис. 3. Как видно, исследуемая зависимость имеет линейный участок на большей части интервала, за исключением узкой окрестности около $h=1$.

Разложение функции $\tilde{\rho}(h)$ в окрестности $h=0$ имеет вид

$$
\tilde{\rho}(h) \approx \frac{\pi h^{2}}{4}\left(1+\frac{h^{2}}{8}+\frac{3 h^{4}}{64}+\frac{25 h^{6}}{1024}\right) .
$$

Разложение плотности в окрестности $h=1$

$$
\begin{aligned}
\tilde{\rho}(h) \approx & 1-\frac{1-h^{2}}{4}\left(1-\frac{3\left(1-h^{2}\right)}{16}\right. \\
& \left.-\left(1+\frac{\left(1-h^{2}\right)}{8}\right) \ln \left(\frac{1-h^{2}}{16}\right)\right)
\end{aligned}
$$

имеет логарифмическую составляющую, характерную для кулоновского потенциала [21]. Вклад логарифмического слагаемого в (29) быстро убывает при $h \rightarrow 1$, что иллюстрирует рис. 4. Точность разложений (28) и (29) иллюстрирует рис. 5.

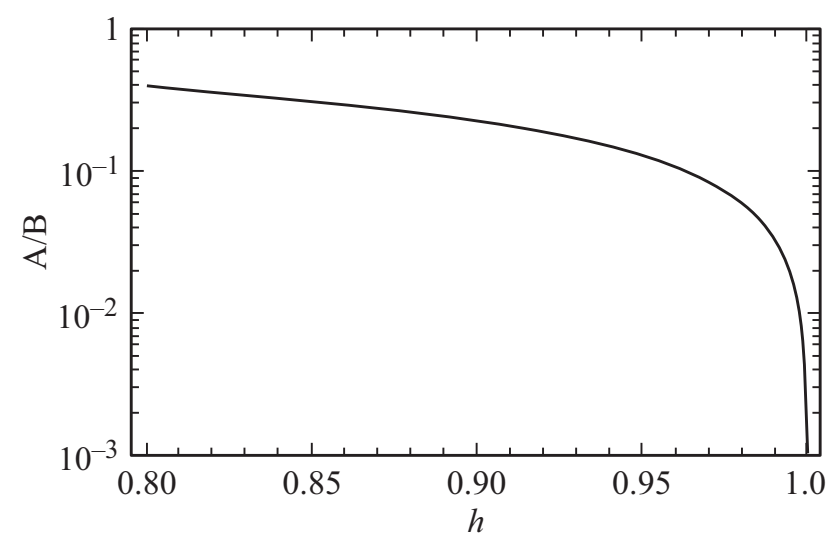

Рис. 4. Зависимость отношения логарифмического слагаемого (А) к слагаемому без логарифма (В) в функции (29) от параметра $h$. Масштаб по оси ординат логарифмический.

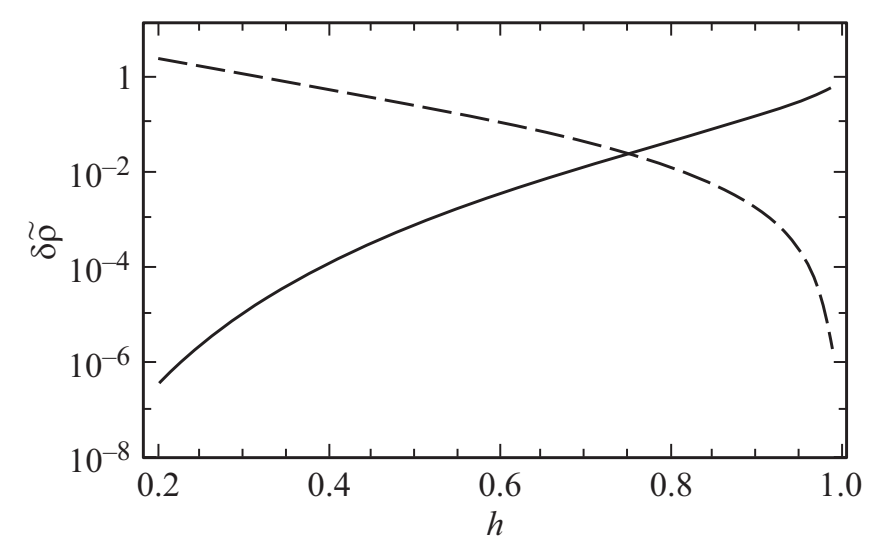

Рис. 5. Относительная ошибка разложений (28) (сплошная линия) и (29) (штриховая линия). Масштаб по оси ординат логарифмический. 


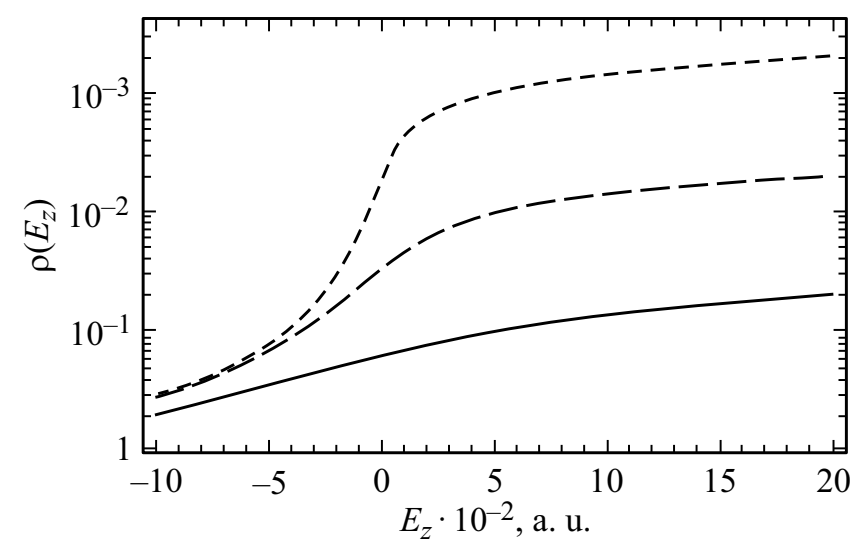

Рис. 6. Зависимость плотности состояний от энергии для полей напряженностью $F=10^{-2}$ (сплошная линия), $10^{-3}$ (штриховая линия, длинные штрихи), $10^{-4}$ а. u. (штриховая линия, короткие штрихи). Масштаб по оси ординат логарифмический.

Подставив разложения (25), (26) и (27) в формулы (20), (28) и (29), можно выразить $\tilde{\rho}(\varepsilon)$ через энергетический параметр. Для всей области положительных энергий с точностью до членов четвертого порядка малости соответствующее приближение имеет вид

$$
\begin{aligned}
& \tilde{\rho}(\varepsilon) \approx 1-\frac{1}{16 \varepsilon^{2}}\left(1-\frac{51}{64 \varepsilon^{2}}\right. \\
& \left.-\left(1-\frac{23}{32 \varepsilon^{2}}\right) \ln \left(\frac{1}{64 \varepsilon^{4}}\left(1-\frac{3}{4 \varepsilon^{2}}\right)\right)\right) .
\end{aligned}
$$

В окрестности нулевого значения энергетического параметра $(|\varepsilon| \ll 1)$ функция $\tilde{\rho}(\varepsilon)$ может быть представлена в виде

$$
\begin{aligned}
& \tilde{\rho}(h) \approx I_{1 / 2}\left(\frac{1}{\sqrt{2}}\right)\left(\frac{1}{2}+h^{2}\right)-\frac{1}{2} I_{-1 / 2}\left(\frac{1}{\sqrt{2}}\right) \\
& -\left(1-h^{2}\right)\left(2 h^{2}-1\right) \frac{\Gamma^{2}(3 / 4)}{\pi} .
\end{aligned}
$$

Таким образом, плотность энергий в большинстве случаев может быть выражена через элементарные функции.

Примеры зависимости плотности состояний от энергии приведены на рис. 6. Наглядно видно сужение ширины области резкой зависимости плотности от энергии при уменьшении напряженности поля.

\section{Заключение}

Энергетический спектр электрона, связанного вблизи поверхности металла полем изображения и запирающим электрическим полем, содержит как непрерывное слагаемое, соответствующее свободному движению параллельно поверхности металла, так и дискретную компоненту, относящуюся к финитному движению перпендикулярно поверхности. Дискретный спектр энергий можно описать в квазиклассическом приближении в широком интервале напряженности. Общий вид спектра характеризуется усредненной характеристикой плотностью спектра энергий. Спектральная плотность может быть выражена через полные эллиптические интегралы, аргумент которых $h$ является сигмоидальной функцией энергетического параметра $\varepsilon=E_{z} / \sqrt{F}$. Ширина переходной области, пропорциональна $F^{-1 / 2}$ и при выключении поля функция $h(\varepsilon)$ переходит в функцию Хевисайда. Нормировка энергии на ширину ступеньки позволяет выделить в плотности состояний приведенную плотность, зависящую только от функции $h$. При значениях энергетического параметра $\varepsilon \geq \sqrt{2}$ плотность спектра описывается моделью асимметричного треугольного потенциала с добавлением логарифмических слагаемых, характерных для потенциалов с кулоновской асимптотикой. Вклад логарифмических слагаемых монотонно убывает с ростом энергии. В области отрицательных значений параметра $\varepsilon \leq-\sqrt{2}$ плотность спектра стремится к плотности ридберговского спектра, что физически соответствует пренебрежению влиянием электрического поля. На интервале $|\varepsilon| \leq \sqrt{2}$, совпадающем по порядку величины с шириной переходной области функции $h$, внешнее электрическое поле и поле изображения вносят сравнимый вклад в энергию электрона. На этом интервале энергетического параметра происходит перестройка от ридберговского спектра к спектру треугольного потенциала. Приведенная плотность всюду, за исключением малой окрестности точки $h=1$, описывается полиномом, содержащим четные степени функции $h$. В окрестности точки $h=1$ действием кулоновского поля на электрон можно пренебречь, и вклад логарифмических слагаемых в плотность стремится к нулю.

В дальнейшем предполагается рассмотреть динамику электрона в условиях конфайнмента под действием ультракоротких лазерных импульсов, которая принципиально зависит от структуры спектра и должна дать генерацию гармоник вторичного излучения, существенно отличающуюся от наблюдаемой для атомов.

\section{Конфликт интересов}

Авторы заявляют, что у них нет конфликта интересов.

\section{Список литературы}

[1] Chulkov E.V., Silkin V.M., Echenique P.M. // Surf. Sci. 1997. V. 391. P. L1217.

[2] Chulkov E.V., Borisov A.G., Gauyacq J.P., Sanchet-Portal D., Silkin V.M., Zhuki V.P., Echenique P.M. // Chem. Rev. 2006. V. 106. P. 4160.

[3] Echenique P.M., Pendry J.B. // Prog. Surf. Sci.1990. V. 32. P. 111.

[4] Fann W.S., Storz R., Bokor J. // Phys. Rev. B. 1991. V. 44. № 19. P. 10980 .

[5] Shumay I.L., Höfer U., Reus Ch., Thomann U., Wallauer W., Fauster Th. // Phys. Rev. B. 1998. V. 58. N 20. P. 13974.

[6] Marks M., Schwalb C.H., Schubert K., Güdde J., HöferU. // Phys. Rev. B. 2011. V. 84. P. 245402. 
[7] Niesner D., Fauster T. // J. Phys. Cond. Matter. 2014. V. 26. P. 393001.

[8] Lin Y., Li Y., Sadowski J.T., Jin W., Dadap J.I., Hybertsen M.S., Osgood R.M. // Phys. Rev. B. 2018. V. 97. P. 165413.

[9] Yildiz D., Kisiel M., Gysin U. et al. // Nat. Mater. 2019. V. 18. P. 1201.

[10] Ge J.-F., Zhang H., He Y., Zhu Z., Yam Y.C., Chen P., Hoffman J.E. // Phys. Rev. B. 2020. V. 101. P. 035152.

[11] Головинский П.А., Преображсенский М.A. // ОПт. и спектр. 2015. Т. 118. № 2. С. 203; Golovinskii P.A., Preobrazhenskii M.A. // Opt. Spectrosc. 2015. V. 118. N 2. P. 191.

[12] Головинский П.А., Преображенский М.A. // Письма в ЖТФ. 2015. Т. 41. № 15. С. 8; Golovinskii P.A., Preobrazhenskii M.A. // Tech. Phys. Lett. 2015. V. 41. N 8. P. 720.

[13] Gao Y., Drake T., Chen Z., DeCamp M.F. // Opt. Lett. 2000. V. 33. N 23. P. 2776.

[14] Salén P., Basini M., Bonetti S., Hebling J., Krasilnikov M., Nikitin A.Y., Shamuilov G., Tibai Z., Zhaunerchuk V., Goryashko V. // Phys. Rep. 2019. V. 836-837. P. 1.

[15] Fröman N., Fröman P.O. Stark Effect in a Hydrogenic Atom or Ion. Imperial College Press. University of Uppsala, Sweden, 2008. 153 p.

[16] Головинский П.А., Преображсенский М.A. // Опт. и спектр. 2017. Т. 122. № 1. С. 133; Golovinskii P.A., Preobrazhenskii M.A. // Opt. Spectrosc. 2017. V. 122. N 1. P. 120.

[17] Головинский П.А., Преображсенский М.A. // Опт. и спектр. 2018. Т. 125. № 3. С. 395; Golovinskii P.A., Preobrazhenskii M.A. // Opt. Spectrosc. 2018. V. 125. N 3. P. 409.

[18] Мигдал А.Б. Качественные методы в квантовой теории. М.: Наука, 1975. 335 с.

[19] Sakurai J.J. Modern Quantum Mechanics. NY:: AdisonWesley, 1994. 500 p.

[20] Градштейн И.С., Рызиик И.М. Таблицы интегралов, рядов и произведений. СПб. БХВ-Петербург, 2011. 1232 с.

[21] Ландау Л.Д., Либшии, Е.М. Квантовая механика. М.: ФИЗМАТЛИТ, 2016. 800 с. 